\title{
COASSIMETRIA E COCURTOSE NA ANÁLISE DOS PREÇOS DAS AÇÕES NO MERCADO FINANCEIRO NACIONAL
}

\author{
CO-SKEWNESS AND CO-KURTOSIS \\ IN THE ANALYSIS OF STOCK PRICING AT IBOVESPA
}

Recebido em 25.04.10 / Aceito em 05.10.10

\begin{abstract}
Alexandre Silva de Oliveira ${ }^{1}$, Luis Felipe Dias Lopes $^{2}$ e Eduardo Botti Abbade ${ }^{3}$
\end{abstract}

\begin{abstract}
Resumo
Este estudo tem como tema de pesquisa a mensuração do preço das ações no mercado financeiro nacional. Como questões, investigam-se qual a influência do terceiro e quarto momentos na precificação de ativos, a influência da coassimetria na correlação da proxy IBOV com as ações, a influência da cocurtose na correlação da proxy IBOV com as ações, a influência conjunta da coassimetria e cocurtose na correlação entre a proxy IBOV e as ações, o seu desempenho comparado com o modelo CAPM e o aumento ou não da precisão. Como método de pesquisa, desenvolveu-se pesquisa bibliográfica e estudo das séries temporais das ações que compunham o índice Ibovespa, em 30 de maio de 2008, tratadas com o uso de regressões múltiplas, tendo como variáveis a volatilidade sistemática, a assimetria sistemática e a curtose sistemática. Como resultados, o trabalho permite afirmar conclusivamente que a coassimetria e a cocurtose não melhoram o desempenho do modelo de precificação de ativos.
\end{abstract}

Palavras-chave: Mercado Financeiro. Preço de Ações. Administração. Cocurtose. Coassimetria.

1 Centro Universitário Franciscano. Doutor em Engenharia Ambiental pela Universidade Federal de Santa Maria. Email: aleoli05@yahoo.com.br

2 Universidade Federal de Santa Maria. Doutor em Engenharia de Produção pela Universidade Federal de Santa Catarina. E-mail: Iflopes@smail.ufsm.br

3 Centro Universitário Franciscano. Mestre em Administração pela Universidade Federal do Paraná. E-mail: eduardo@unifra.br 


\begin{abstract}
The central issue of this research is to investigate and measuring the stock price in the brazilian financial market. It was investigate the influence of the third and fourth time in the pricing of assets, the influence of co-skewness in correlation with the proxy IBOV stocks, the influence of co-kurtosis in correlation with the proxy IBOV stocks, the influence of co-skewness and co-kurtosis in the correlation between the proxy IBOV and stocks, its performance compared with the CAPM and the increase of the accuracy. Is was developed literature research and study of time series of the stocks that constitutes the Ibovespa index on 30 May 2008, analyzed with the use of multiple regressions with the variables to systematic volatility, the systematic skewness and systematic kurtosis. As a result it was observed conclusively that co-skewness and co-kurtosis do not improve the performance of the model of pricing of assets.
\end{abstract}

Keywords: Financial Market. Stock Pricing. Business. Co-skewness. Co-kurtosis.

\title{
INTRODUÇÃO
}

Desde suas origens, os preços de negociação das ações tentam ser estimadas no mercado financeiro. A negociação de ativos vem sofrendo modificações com a inserção de sistemas eletrônicos de solicitação de compra e venda, tornando os mercados mais dinâmicos. Todas essas mudanças têm permitido o crescimento e desenvolvimento desses mercados, fornecendoIhes maior liquidez e capacidade de cumprir com sua função. A função básica pela quais investidores buscam o mercado financeiro é a manutenção da liquidez dos recursos, casada como a maximização da remuneração dos ativos.

Dada a relevância da estimativa do preço das ações para atendimento a esse fim, este projeto de estudo apresenta uma proposta de mensuração do preço das ações no mercado financeiro nacional. Em função do exposto, pretende-se verificar se existe significância à introdução do terceiro e quarto momento, conhecidos como coassimetria e cocurtose ao tradicional modelo de precificação de ativos.

No momento da compra ou venda de uma ação, verificar se o preço é justo para o mercado, se este está sobre ou subvalorizado se faz necessário. O modelo de apreçamento de ativos Capital Asset Price Model (CAPM), proposto por Sharpe (1964), é o modelo mais comumente utilizado para determinar o valor das ações. Porém, evidências apontam ineficiência nesta mensuração. Estudos em vários mercados estão sendo desenvolvidos para identificar a influência da assimetria e da curtose neste modelo. No mercado brasileiro, no entanto, não foi identificado nenhum estudo buscando evidenciar esta tendência. Dada essa lacuna existente, somada ao fato de que o preço de ativos brasileiros pode apresentar características peculiares e diferentes das dos outros países, toma-se como problema proposto para estudo a seguinte questão: Como mensurar o preço das ações no mercado financeiro nacional?

O objetivo geral deste trabalho é mensurar o preço das ações no mercado financeiro nacional. Os objetivos específicos são: (1) aplicar o terceiro e quarto momento na precificação de ativos; (2) verificar a influência da coassimetria na correlação da proxy IBOV com as ações; (3) verificar a influência da cocurtose na correlação da proxy IBOV com as ações; (4) verificar a influência conjunta da coassimetria e cocurtose na correlação entre a proxy IBOV e as ações; (5) comparar com o tradicional modelo CAPM; e (6) verificar o aumento ou não da precisão. 
O modelo CAPM tem sérias dificuldades para explicar a performance passada da maioria das ações do mercado financeiro. Esta particular teoria de trabalho restringe o risco-retorno a uma relação simples variância-média e/ou a uma função utilitária quadrática. Porém empíricas evidências mostram que a hipótese de normalidade tem sido rejeitada por muitos retornos de ações. Além do mais, a utilização de uma função quadrática por um investidor implica em um incremento a aversão ao risco. Ao invés disso, é mais razoável assumir que a aversão ao risco decresce com um incremento do tempo. Neste trabalho, serão consideradas algumas extensões do tradicional modelo CAPM, que conta com momentos condicionais mais fortes e uma maior variedade estrutural do conceito de prêmio pelo risco. Em particular, será examinado o papel da coassimetria e da cocurtose na precificação de ativos.

Neste estágio, a pesquisa investigou se a assimetria e a curtose têm alguma relevância na explanação dos retornos dos ativos. Assimetria caracteriza o grau da não normalidade da distribuição em volta da média. Positiva (negativa) assimetria indica uma anormalidade positiva ou negativa da distribuição. Curtose forte ou fraca indica uma distribuição mais íngreme ou achatada. A assimetria e a curtose podem ser conjuntamente analisadas na referência do mercado. Similarmente como o desvio-padrão ou o beta da carteira é chamado de risco sistemático na precificação de ativos, pode ser examinado se existe uma assimetria e curtose sistemática, ambas conhecidas como coassimetria e cocurtose, de acordo com Christie-David e Chaudry (2001).

Provando-se que existe uma assimetria positiva dos retornos, investidores irão preferir ativos com negativa coassimetria. A cocurtose mensura a probabilidade dos retornos extremos ocorrerem simultaneamente em um dado ativo e no mercado. As características comuns dos modelos contabilizados pela coassimetria e a cocurtose é incorporar fortes momentos no trabaIho de precificação de ativos.

É comum encontrar, na literatura, a investigação do terceiro e do quarto momento CAPM. A especificação teórica do terceiro momento CAPM é desenvolvida por Kraus e Litzenberger (1976), Ingersoll (1975), Gamba e Rossi (1998), Jurcenzko e Maillet (2002) e por Ranaldo e Favre (2003). Harvey e Siddique (2000) encontraram que a sistemática assimetria requer uma média anual de prêmio pelo risco de 3,6\% para as ações dos Estados Unidos. Eles também encontraram que os portfólios com forte assimetria sistemática são compostos por ações de destaque. Demonstraram ainda que a assimetria, a cocurtose e a curtose são precificadas em mercados emergentes individuais, mas não em mercados desenvolvidos, o que é mais uma evidência que instiga a aplicação do modelo ao mercado brasileiro. Eles observaram que a volatilidade e o retorno em mercados emergentes são positivamente relacionados de forma significativa. Mas eles desaparecem quando a coassimetria, assimetria e curtose são consideradas. Harvey (2000) acredita que a causa para isso é o baixo grau de integração dos mercados emergentes. Hwang e Satchell (1999), Chung, Johnson e Schill (2001) e Berenyi (2002) propuseram o uso do modelo cúbico como um teste para a coassimetria e a cocurtose. Berenyi (2002) aplicou o quarto momento ao estudo de fundos de investimento. Christie-David e Chaudry (2001) empregaram o quarto momento CAPM em mercados futuros.

Hwang e Satchell (1999) investigaram a coassimetria e a cocurtose em mercados emergentes e demonstraram que a curtose sistemática explica melhor os retornos dos mercados emergentes do que a assimetria sistemática.

Este estudo investigou a contribuição dos terceiro e quarto momentos na precificação das ações brasileiras. 


\section{COASSIMETRIA E E COCURTOSE}

Esta seção busca apresentar o referencial teórico utilizado inicialmente como suporte ao tema da pesquisa. Trata-se de uma revisão da literatura disponível, a fim de se tomar conhecimento do que já existe sobre o assunto e oferecer contextualização e consistência à investigação.

\subsection{A existência da coassimetria e da cocurtose no mercado financeiro}

A existência da coassimetria e a cocurtose na distribuição dos retornos é conhecida em alguns mercados. A pesquisa sobre elas no retorno na distribuição de retornos é duplamente essencial. Por um lado, os padrões de distribuição de retorno podem ser originários das estratégias específicas dos traders. Os administradores de ativos procuram uma variedade de ativos e estratégias que gerem perfis de pay-offs extremamente diferentes dos ativos tradicionais. Por outro lado, a inclinação e achatamento da distribuição de retornos podem ser vistas como uma expressão estatística da ineficiência de mercado. Especialmente, a não-normalidade do retorno das distribuições pode ser devido à iliquidez, à falta de divisibilidade e ao baixo nível de transparência das informações. Todos estes fatores contrastam com a suposição de que o modelo CAPM suporta essas variáveis. Mediante a revisão de literatura, será apresentado o modelo CAPM, como e por que esses fatores representam dados elegíveis da coassimetria e da cocurtose entre as ações dos mercados.

\subsection{O modelo de precificação de ativos}

O modelo de risco e retorno ainda usado e que é o padrão na maior parte das análises de finanças é o modelo de precificação de ativos de capital, dado pela equação:

$$
E\left(R_{j}\right)-R_{f}=\beta_{j}\left[E\left(R_{m}\right)-R_{f}\right]
$$

em que:

$E\left(R_{j}\right)=$ retorno esperado da ação $j$;

$R_{f}=$ ativo sem risco: é aquele no qual o retorno real é sempre o esperado (como exemplo, cita-se o Título do Tesouro Nacional);

$E\left(R_{m}\right)=$ retorno esperado do mercado $m$;

$\hat{a}_{j}=$ beta da ação $j$.

O modelo é sustentado pelas seguintes premissas: (1) supõe que não existem custos de transações; (2) que todos os títulos são negociáveis e são infinitamente divisíveis; e (3) ao tomarem decisões sobre suas carteiras, os investidores o fazem levando em conta apenas as condições de risco e retorno.

Um dos aspectos tem sido a forma de como devem ser relacionados o risco e o retorno de um título. O CAPM, entre outros modelos, consegue dimensionar esses dois componentes e seus reflexos sobre a taxa de retorno esperada de um título, conforme Ross, Westerfield e Jaffe 
(1995). Na teoria, a preocupação com o cálculo de um ativo, conhecido como valor intrínseco ou valor justo, tem sido constante.

A determinação desses dois componentes, risco e retorno, é também uma das tarefas primordiais dos investidores; e o resultado dessa mensuração é ingrediente crucial na construção e formação das carteiras de títulos.

A dificuldade em medir esses componentes, risco e retorno, pode ser entendida se for imaginado um investidor tentando delinear cada evento possível (preço de uma ação, por exemplo) e estimar a sua probabilidade de ocorrência e o efeito de cada um desses preços sobre suas alternativas de investimento. Isso seria impraticável. Na realidade, isso pode ser evitado se os retornos médios ou retornos esperados forem diretamente estimados e a seu lado, a divergência provável de cada retorno com relação a sua média ou ao seu valor esperado. Dessa forma, será utilizada a média ponderada como retorno esperado e os desvios dessa média (variância e desvio padrão).

A maior vantagem do modelo de avaliação de ativos (CAPM) está em que ele considera a volatilidade, permitindo estudar o impacto duplo e simultâneo da lucratividade e do risco sobre a ação. Além dos pressupostos do mercado eficiente, o modelo pressupõe também que o investidor é avesso ao risco e se utiliza dos conceitos de média e variância na escolha das alternativas. Dessa forma, todo investidor pode ser caracterizado pelo maior ou menor grau de aversão ao risco, de tal modo que existem investidores neutros em relação ao risco, aqueles investidores avessos ao risco e aqueles investidores que preferem o risco a qualquer alternativa.

O retorno excedente de uma aplicação em ativo, acima de uma aplicação livre de risco (título do Tesouro Nacional), é considerado como recompensa pelo risco assumido, chamado "prêmio por risco". Na equação do CAPM, visto em [1] o beta de ação ( $\left.\hat{a}_{j}\right)$ é dado por:

$$
\beta_{j}=\frac{\operatorname{Cov}\left(R_{j}-R_{m}\right)}{\sigma^{2} R_{m}} .
$$

O beta na fórmula acima, de acordo com Gropelli e Nikbakht (2005), é definido como a covariância do título dividida pela variância da carteira de títulos do mercado (Índice BOVESPA), que é a medida de volatilidade dos retornos dos títulos com relação aos retornos do mercado como um todo, partindo do princípio de que todos os títulos tendem a ter os seus preços alterados com maior ou menor proporção às alterações do mercado como um todo.

Em equilíbrio, todos os ativos com risco devem cair ao longo da linha reta, conhecida como a reta de mercados de títulos. Assim, o beta será igual a um $\left(\hat{a}_{R m}=1\right)$, isto porque a covariância do mercado é igual à variância do mercado, ou seja, uma carteira média tem beta igual a 1,0 em relação a ela mesma. Um título com beta $=1$ é considerado neutro. À medida que o mercado como um todo suba hipoteticamente 3\% (Índice BOVESPA), aquele título tende a subir também $3 \%$. À medida que o mercado como um todo (Índice BOVESPA) cai hipoteticamente 5\%, aquele título tenderá a cair 5\%.

Um título com beta $>1,0$, por exemplo: uma ação com $\hat{a}=1,15$ significa que se o mercado (Índice BOVESPA) como um todo apresentar uma queda de 10\%, aquela ação deverá sofrer uma baixa de 11,50\% no seu preço. Um título com beta $<1,0$, por exemplo: uma ação com $\hat{a}=0,5$ significa que se o mercado cair $6 \%$, a ação deverá sofrer uma baixa de somente $3 \%$.

É importante lembrar-se de que o retorno esperado e, portanto, o prêmio de risco de um ativo depende apenas do risco sistemático. Como ativos com betas maiores têm riscos sistemáticos mais altos, têm também retornos esperados maiores. Dessa forma, conhecendo-se as características 
de risco (beta) de uma ação, é possível estimar-se o preço justo (ou valor intrínseco), tendo-se a indicação se o título é ou não uma boa opção de compra.

\subsection{A introdução de mais momentos no modelo}

É a ênfase das estratégias de mercado aplicadas por administradores de ativos que geram típicas tendências de mercado, conforme Christie-David e Chaudry (2001). A sensibilidade dos traders aos acontecimentos globais gera comportamentos que influenciam nas tendências de alta ou baixa no retorno dos ativos.

A iliquidez de ativos, segundo Gamba e Rossi (1997), também faz com que o volume de negociações em uma imediata execução aconteça sem que impacte sobre o preço, como é o que acontece com mercado emergente. Caso aconteça algo no mercado americano, instantaneamente o preço das ações no mercado brasileiro sofre altas variações devido à baixa liquidez dos ativos negociados. A iliquidez dos ativos é uma das principais suposições do modelo CAPM. Um baixo nível de liquidez exige que exista um prêmio. Lo (2001) derivou o prêmio pela liquidez ao assumir que a autocorrelação é uma proxy para os custos de iliquidez.

Outra suposição atrás do CAPM é que os ativos são infinitamente divisíveis (BERENYI, 2002). Isso pressupõe que o investidor irá escolher um determinado tipo de ativo, independentemente do tamanho do investimento.

E a ineficiência de mercado pode ser também devido à opaca ou assimétrica informação. O baixo grau de transparência das informações é particularmente justificado pela pequena posição e arbítrio de estratégias empreendidas pelos administradores de ativos. Este tipo de estratégia de mercado implica em uma posição disfarçada, especialmente em mercados ilíquidos. De fato, uma completa e transparente informação revela e arrisca oportunidades de negócios.

\subsubsection{Coassimetria e a cocurtose}

De maneira a incluir a influência da assimetria do mercado sobre o modelo de precificação de ativos, Rubinstein (1973) e Kraus e Lintzenberger (1976) desenvolveram o CAPM com o terceiro momento. A hipótese básica que sustenta as ideias dos autores é que a distribuição de frequência das taxas de retorno não são simétricas, o que induziria a preferência dos investidores por assimetria negativa. Um modelo de precificação coerente com as ideias de Kraus e Lintzenberger, que inclui o terceiro momento, difere do modelo tradicional do CAPM pela adição da terceira parte da equação, razão entre a covariância dos retornos do portfólio com o quadrado do retorno do mercado e o terceiro momento em torno da média do mercado, que multiplica o quadrado do retorno do mercado.

A coassimetria e a cocurtose são representadas pela introdução do terceiro e do quarto momento CAPM (LIM, 1989). O quarto momento é acrescentado para incorporar os efeitos da curtose (FANG; LAI, 1997). Assim, há a inclusão da quarta parte ou da razão entre a covariância do retorno do portfólio com o cubo do retorno do mercado pelo quarto momento em torno da média do mercado. Estes representam um modelo de precificação com beta, assimetria e curtose sistemática. Neste modelo, $i$ denota uma ação genérica e $m$ o mercado de referência, ou seja, o mercado brasileiro. $R_{i}$ e $R_{m}$ denotam os respectivos retornos. O problema de investimento de um investidor é maximizar a expectativa de ganhos no final do período. $\mathrm{O}$ investidor espera que o ganho possa ser representado, estatisticamente, pelos terceiro e quarto momentos padronizados, respectivamente, para o cubo da volatilidade e a volatilidade elevada na quarta (CATARINA; CERETTA; MULLER, 2007). 


$$
E\left(R_{i}\right)-R_{f}=\alpha_{1} \beta_{i, m}+\alpha_{2} S_{i, m}+\alpha_{3} K_{i, m}
$$

com:

Risco Sistemático:

$$
\beta_{i, m}=\frac{E\left[\left(R_{i}-\bar{R}_{i}\right)\left(R_{m}-\bar{R}_{m}\right)\right]}{E\left(\left(R_{m}-\bar{R}_{m}\right)^{2}\right)} .
$$

Sistemática Assimetria:

$$
S_{i, m}=\frac{E\left[\left(R_{i}-\bar{R}_{i}\right)\left(R_{m}-\bar{R}_{m}\right)^{2}\right]}{E\left(\left(R_{m}-\bar{R}_{m}\right)^{3}\right)} .
$$

Sistemática Curtose:

$$
K_{i, m}=\frac{E\left[\left(R_{i}-\bar{R}_{i}\right)\left(R_{m}-\bar{R}_{m}\right)^{3}\right]}{E\left(\left(R_{m}-\bar{R}_{m}\right)^{4}\right)} .
$$

Assim, $\mathrm{R}_{\mathrm{i}}$ é o retorno do ativo, $\bar{R}_{i}$ é a expectativa de retorno do ativo $i, R_{m}$ é o retorno do mercado, $\bar{R}_{m}$ é a expectativa de retorno do mercado $m$. $S$ é o terceiro momento e $K$ é o quarto momento.

Já os valores $a_{1} a_{2}$ e $a_{3}$ (HARVEY; SIDDIQUE, 2000) são, respectivamente, o incremento pelo risco $\hat{a}$, o decréscimo em função da assimetria sistemática $S$ e o incremento em função da curtose sistemática, $K$. Estes três alfas são dados por:

$$
\begin{gathered}
\alpha_{1}=\frac{d E\left(R_{i}\right)}{d \sigma^{2}\left(R_{i}\right)} \sigma^{2}\left(R_{m}\right)=\left(R_{m, t}-R_{f, i}\right), \\
\alpha_{2}=\frac{d E\left(R_{i}\right)}{d S^{3}\left(R_{i}\right)} S^{3}\left(R_{m}\right)=\left(R_{m, t}-E\left(R_{m}\right)\right)^{2} \\
\alpha_{3}=\frac{d E\left(R_{i}\right)}{d \sigma^{2}\left(R_{i}\right)} K^{4}\left(R_{m}\right)=\left(R_{m, t}-E\left(R_{m}\right)\right)^{3} .
\end{gathered}
$$


E, assim, o modelo cúbico ou de quarto momento do CAPM fica:

$$
E\left(R_{i}\right)-R_{f}=\alpha+\beta_{i, m}\left(R_{m, t}-R_{f, i}\right)+S_{i, m}\left(R_{m, t}-E\left(R_{m}\right)\right)^{2}+K_{i, m}\left(R_{m, t}-E\left(R_{m}\right)\right)^{3} .
$$

em que $\hat{a}_{i, m}$ é uma proxy do risco sistemático, $S_{i, m}$ é uma proxy da assimetria sistemática e $K_{i, m}$ é uma proxy da curtose sistemática.

Fang e Lai (1997) demonstraram que, na presença de curtose, a taxa de retorno esperada em excesso deverá estar relacionada não somente com a variância sistemática e assimetria sistemática, mas também com a curtose sistemática. Portanto, o retorno esperado em excesso deverá ser maior com o aumento da variância e da curtose sistemática e, de forma contrária, deverá ser menor com o aumento da assimetria sistemática.

Nos estudos anteriores, Kraus e Litzenberger (1976), ao testarem o modelo CAPM com a inclusão do terceiro momento, confirmaram a arguição de Arditi (1967) de que a aversão ao risco diminui com o aumento da riqueza, ou seja, o prêmio pela assimetria tem sinal oposto à assimetria do mercado. Lim (1989) obteve resultados que sustentam a ideia de que os investidores preferem a coassimetria quando as taxas de retorno do mercado apresentam assimetria positiva e são avessos à coassimetria quando as taxas de retorno possuem assimetria negativa.

Fang e Lai (1997) incorporaram os efeitos da assimetria e da curtose no modelo de precificação de ativos ao estudarem 27 portfólios compostos por ações listadas na New York Stock Exchange (NYSE), que abrangeu três subperíodos de tempo, compreendidos entre 1974 e 1988. Os resultados sustentam a afirmação de que a assimetria e a curtose estão relacionadas com o retorno, e não somente a variância sistemática.

Cunhachinda et al. (1997) ao analisarem a assimetria obtiveram como resultados que a incorporação do terceiro momento no processo de precificação dos ativos gera uma grande alteração na construção do mesmo e que os investidores trocam retorno esperado por assimetria. Estes resultados são condizentes com os de Peiró (1999), que demonstrou que a preferência por assimetria é um fator de grande importância e não deve ser ignorada no processo de avaliação de ativos arriscados.

Harvey e Siddique (2000), ao estudarem os efeitos da assimetria, obtiveram como resultados, ao analisar portfólios de ações da NYSE, que a coassimetria é importante e ajuda a explicar a variação contemporânea dos retornos dos ativos incorporando aos mesmos um prêmio de $3,6 \%$ ao ano.

A seguir é apresentada a metodologia utilizada para a realização do trabalho, os resultados obtidos e as conclusões.

\section{METODOLOGIA}

Nesta seção estão apresentadas as características do estudo proposto, bem como os procedimentos que foram efetuados para o teste das hipóteses pré-estabelecidas. 


\subsection{Classificação da pesquisa}

Vergara (2000) apresentou uma taxonomia para a tipificação de pesquisas, considerando basicamente dois aspectos: fins e meios. Tomando-se por base esta classificação, pode-se dizer que a pesquisa proposta será descritiva quanto aos fins e documental e de séries temporais quanto aos meios. Descritiva porque buscará expor algumas características do mercado à vista financeiro do Brasil, estabelecendo correlações entre variáveis. Documental e de análise de séries temporais, pois utilizará materiais publicados em livros, revistas, jornais, redes eletrônicas, registros, anais, a fim de dar suporte ao referencial teórico, e utilizará os dados reais passados das ações que compõem o índice Bovespa.

\subsection{Série temporal}

A série temporal será composta pelo preço de fechamento das 53 ações de maior liquidez da Bolsa de Valores de São Paulo e que compunham o índice Bovespa em dezembro de 2006.

Conta com a análise de dados diários de 19 de fevereiro de 1992 até 30 de maio de 2008.

\subsection{Tratamento dos dados}

Análise quantitativa, estatística, com o uso de modelos de regressão múltipla, uso do teste de significância $t$ de Student, do teste de significância $f$ de Snedecor para a regressão e do valor $p$. E análise do Coeficiente de Determinação Ajustado $R^{2}$ Ajustado.

Para o teste da hipótese nula, de acordo com Sartoris (2003), é calculado o F estatístico [11]:

$$
F=\frac{S S E_{0}-S S E}{S S E} \frac{N-2 p-1}{p}
$$

Em [11], SSE $E_{0}$ denota o somatório do quadrado do resíduo do modelo de regressão restringido por $\hat{a}_{i}=0(i=1, \ldots ., p)$; SSE é o somatório do quadrado dos resíduos da equação irrestrita, e $N$ é o número de observações. As estatísticas ao final da equação são assintoticamente a distribuição $F$ dentro do assunto não-causalidade, com $p$ graus de liberdade no denominador e $(N-2 p-1)$ graus de liberdade no numerador.

\subsection{Hipóteses}

O estudo focará basicamente seis hipóteses para a mensuração do preço das ações. A primeira, a hipótese 1, pressupõe que tanto a coassimetria como a cocurtose não são significativas. Esta hipótese será testada contra a hipótese alternativa de que eles apresentam, sistematicamente, significância, hipótese 2.

- Hipótese um (H1): não há uma melhoria na precificação das ações brasileiras com o uso da coassimetria e da cocurtose. Este valor será obtido com o cálculo do teste $f, \operatorname{com} f$ menor que 1,96; 
- Hipótese dois $\left(\mathrm{H}_{2}\right)$ : há significância estatística da coassimetria e da cocurtose, medido com o teste de significância $f$, com f maior que 1,96;

- Hipótese três $\left(\mathrm{H}_{3}\right)$ : não há significância estatística da coassimetria, medida com o teste de significância $t$ de Student, com $t$ menor que 1,96;

- Hipótese quatro $\left(\mathrm{H}_{4}\right)$ : há significância estatística da coassimetria, medida com o teste de significância $t$ de Student, com $t$ maior que 1,96;

- Hipótese cinco $\left(\mathrm{H}_{5}\right)$ : nNão há significância estatística da cocurtose, medida com o teste de significância $t$ de Student, com $t$ menor que 1,96;

- Hipótese seis $\left(\mathrm{H}_{6}\right)$ : Há significância estatística da cocurtose, medida com o teste de significância $t$ de Student, com $t$ maior que 1,96.

\section{RESULTADOS E DISCUSSÃO}

A análise dos resultados está dividida em três seções, que são: apresentação das ações que compõem o índice Bovespa, análise descritiva das variáveis, análise da estacionariedade das variáveis, medidas de dispersão das ações, coeficientes estimados no relacionamento entre retorno esperado e suas medidas de dispersão, coeficientes estimados para os modelos de mercado no período estudado e resumo dos resultados obtidos.

\subsection{Carteira do Ibovespa}

Como pode ser observado na tabela 1, na carteira do Ibovespa, em 30 de maio de 2008, somente 10 papéis são responsáveis por mais 50\% da participação do índice Ibovespa: PETR4, VALE5, BBDC4, VALE3, ITAU4, USIM5, UBBR11, CSNA3, PETR3 e GGBR4. E mais de 50\% dos papéis que compõem o índice possuem participação menor do que $1 \%$. A maior concentração de ações é do tipo preferencial - 40 papéis, e 27 papéis do tipo ordinária.

Tabela 1 - Carteira do Ibovespa em maio de 2008

continua...

\begin{tabular}{l|l|l|r|r}
\hline \multicolumn{1}{c|}{ Código } & \multicolumn{1}{c|}{ Ação } & \multicolumn{1}{c|}{ Tipo } & \multicolumn{1}{c}{ Qtde. Teórica (1) } & \multicolumn{1}{c}{ Part.(\%) (2) } \\
\hline PETR4 & PETROBRAS & PN EB & 227,391125 & 14,139 \\
\hline VALE5 & VALE R DOCE & PNA & 161,4543183 & 12,749 \\
\hline BBDC4 & BRADESCO & PN & 68,23067052 & 3,84 \\
\hline VALE3 & VALE R DOCE & ON & 34,62299612 & 3,349 \\
\hline ITAU4 & ITAUBANCO & PN ED & 45,2462966 & 3,161 \\
\hline USIM5 & USIMINAS & PNA EB & 25,61171334 & 3,063 \\
\hline UBBR11 & UNIBANCO & UNT & 76,2178941 & 2,782 \\
\hline CSNA3 & SID NACIONAL & ON EDJ & 25,80752007 & 2,768 \\
\hline PETR3 & PETROBRAS & ON EB & 35,41578254 & 2,64 \\
\hline GGBR4 & GERDAU & PN & 26,73803838 & 2,591 \\
\hline ITSA4 & ITAUSA & PN EBS & 151,0715182 & 2,437 \\
\hline BBAS3 & BRASIL & ON & 55,86664783 & 2,379
\end{tabular}


Conclusão

\begin{tabular}{|c|c|c|c|c|}
\hline Código & Ação & Tipo & Qtde. Teórica (1) & Part.(\%) (2) \\
\hline CMIG4 & CEMIG & PN EDB & 35,41870664 & 1,8 \\
\hline ALLL11 & ALL AMER LAT & UNT ED & 54,52412231 & 1,743 \\
\hline NETC4 & NET & $\mathrm{PN}$ & 46,33724001 & 1,562 \\
\hline CESP6 & CESP & PNB & 39,87783845 & 1,545 \\
\hline TNLP4 & TELEMAR & $\mathrm{PN}$ & 27,16035816 & 1,521 \\
\hline BRAP4 & BRADESPAR & PN EJ & 19,54667734 & 1,415 \\
\hline CYRE3 & CYRELA REALT & ON ED & 31,29976703 & 1,279 \\
\hline GOLL4 & $\mathrm{GOL}$ & PN ED & 31,85457404 & 1,228 \\
\hline AMBV4 & AMBEV & PN ES & 6,62864702 & 1,211 \\
\hline BTOW3 & B2W VAREJO & ON & 14,03879631 & 1,14 \\
\hline TAMM4 & TAM S/A & PN & 20,08483372 & 1,13 \\
\hline LAME4 & LOJAS AMERIC & $\mathrm{PN}$ & 64,14457876 & 1,125 \\
\hline PRGA3 & PERDIGAO S/A & ON EJ & 16,38570409 & 1,105 \\
\hline ELET6 & ELETROBRAS & PNB & 28,43395425 & 1,081 \\
\hline LREN3 & LOJAS RENNER & ON & 18,55979692 & 1,072 \\
\hline CSAN3 & COSAN & $\mathrm{ON}$ & 23,79935057 & 1,048 \\
\hline SDIA4 & SADIA S/A & $\mathrm{PN}$ & 55,9152539 & 1 \\
\hline TCSL4 & TIM PART S/A & $\mathrm{PN}$ & 119,1643288 & 0,994 \\
\hline GFSA3 & GAFISA & ON & 18,45615393 & 0,992 \\
\hline ELET3 & ELETROBRAS & $\mathrm{ON}$ & 25,17188522 & 0,927 \\
\hline NATU3 & NATURA & ON & 31,13806876 & 0,89 \\
\hline BRKM5 & BRASKEM & PNA & 41,21479198 & 0,87 \\
\hline VIVO4 & VIVO & $\mathrm{PN}$ & 48,3476949 & 0,862 \\
\hline ARCZ6 & ARACRUZ & PNB & 42,77539835 & 0,845 \\
\hline ELPL6 & ELETROPAULO & PNB ED & 15,30948824 & 0,83 \\
\hline CPLE6 & COPEL & PNB & 18,61022301 & 0,821 \\
\hline GOAU4 & GERDAU MET & $\mathrm{PN}$ & 6,233958044 & 0,813 \\
\hline DURA4 & DURATEX & $\mathrm{PN}$ & 16,00030627 & 0,794 \\
\hline CCRO3 & CCR RODOVIAS & ON & 16,8093432 & 0,793 \\
\hline EMBR3 & EMBRAER & $\mathrm{ON}$ & 30,98085482 & 0,792 \\
\hline BRTO4 & BRASIL TELEC & $\mathrm{PN}$ & 25,83070614 & 0,761 \\
\hline BRTP4 & BRASIL T PAR & $\mathrm{PN}$ & 19,06464423 & 0,733 \\
\hline PCAR4 & P.ACUCAR -CBD & PN ED & 13,11991143 & 0,73 \\
\hline VCPA4 & VCP & $\mathrm{PN}$ & 9,044711135 & 0,704 \\
\hline CPFE3 & CPFL ENERGIA & $\mathrm{ON}$ & 11,05947602 & 0,684 \\
\hline TNLP3 & TELEMAR & $\mathrm{ON}$ & 8,472841676 & 0,637 \\
\hline JBSS3 & JBS & ON ED & 47,52360665 & 0,605 \\
\hline USIM3 & USIMINAS & ON EB & 4,476446298 & 0,561 \\
\hline RSID3 & ROSSI RESID & $\mathrm{ON}$ & 22,36230097 & 0,541 \\
\hline SBSP3 & SABESP & $\mathrm{ON}$ & 8,810235849 & 0,54 \\
\hline CRUZ3 & SOUZA CRUZ & ON EJ & 7,634776262 & 0,524 \\
\hline UGPA4 & ULTRAPAR & $\mathrm{PN}$ & 5,567425756 & 0,479 \\
\hline KLBN4 & KLABIN S/A & $\mathrm{PN}$ & 49,91545486 & 0,478 \\
\hline BNCA3 & NOSSA CAIXA & ON & 11,32103965 & 0,424 \\
\hline TRPL4 & TRAN PAULIST & $\mathrm{PN}$ & 5,373314619 & 0,344 \\
\hline BRTP3 & BRASIL T PAR & $\mathrm{ON}$ & 4,279585188 & 0,34 \\
\hline TCSL3 & TIM PART S/A & $\mathrm{ON}$ & 29,55155814 & 0,333 \\
\hline LIGT3 & LIGHT S/A & ON & 7,592320742 & 0,28 \\
\hline TMAR5 & TELEMAR N L & PNA & 1,87623517 & 0,265 \\
\hline TLPP4 & TELESP & $\mathrm{PN}$ & 4,023747722 & 0,26 \\
\hline CGAS5 & COMGAS & PNA & 2,712434722 & 0,186 \\
\hline TMCP4 & TELEMIG PART & $\mathrm{PN}$ & 2,206451948 & 0,186 \\
\hline CLSC6 & CELESC & PNB ED & 2,680479095 & 0,182 \\
\hline CCPR3 & CYRE COM-CCP & ON ED & 6,259953405 & 0,098 \\
\hline \multicolumn{3}{|c|}{ Quantidade Teórica Total } & $2.208,66$ & 100 \\
\hline
\end{tabular}


Essa constatação mostra que, em sua maioria, os papéis mais líquidos são os de ações prefernciais que possuem preferência nos dividendos, mas que não dão poder dentro das corporações, como as ações ordinárias, o que indica uma preferência pelo mercado em atuar de forma especulativa e não com o intuito de obter poder dentro das empresas.

Entre as dez corporações de maior participação no índice Ibovespa, que representa mais de $80 \%$ da liquidez dos papéis negociados na bolsa de São Paulo, estão empresas ligadas ao setor de minas e energia, siderurgia e metalurgia e instituições financeiras. A Petrobrás, Vale e Usiminas do setor de minas e energia; a Siderúrgica Nacional e a Gerdau do setor de siderurgia e metalurgia; e o Bradesco, Itaú e Unibanco do setor financeiro.

\subsection{Estatística descritiva das variáveis}

Na tabela 2 pode-se observar a análise descritiva da variável retorno das ações que compõem o índice Bovespa. São apresentadas as médias, variâncias, assimetrias e curtoses. As variáveis possuem em sua totalidade característica platicúrtica, curtose maior que 0,263 , portanto, com caudas longas e achatadas.

Tabela 2 - Estatística descritiva das variáveis das ações

\begin{tabular}{|c|r|r|r|r|}
\hline \multirow{2}{*}{ Ação } & \multicolumn{5}{|c|}{ Parâmetros da Proxy de mercado e das ações estudadas } \\
\cline { 2 - 6 } & \multicolumn{1}{|c|}{$\boldsymbol{\mu}$} & \multicolumn{1}{c|}{$\boldsymbol{\sigma}^{\mathbf{2}}$} & \multicolumn{1}{c|}{$\boldsymbol{S}$} & \multicolumn{1}{c|}{$\boldsymbol{\boldsymbol { K }}$} \\
\hline IBOV & 0,000914 & 0,022156 & 0,349850 & 14,261690 \\
\hline PETR4 & 0,002300 & 0,032700 & $-0,535000$ & 72,560000 \\
\hline VALE5 & 0,001300 & 0,000700 & 1,152000 & 17,430000 \\
\hline BBDC4 & 0,001100 & 0,000700 & $-0,178000$ & 4,710000 \\
\hline VALE3 & 0,001200 & 0,000700 & 0,389000 & 9,200000 \\
\hline ITAU4 & 0,001248 & 0,000629 & 0,113255 & 2,579767 \\
\hline USIM5 & 0,001114 & 0,001027 & $-0,068101$ & 2,077652 \\
\hline UBBR11 & 0,000747 & 0,000757 & $-0,014746$ & 2,225836 \\
\hline CSNA3 & 0,001731 & 0,019097 & 2,399461 & 0,000794 \\
\hline PETR3 & 0,001449 & 0,000950 & $-3,694463$ & 84,177970 \\
\hline GGBR4 & 0,001400 & 0,000700 & $-0,239000$ & 7,670000 \\
\hline ITSA4 & 0,001159 & 0,000639 & $-0,023031$ & 4,742675 \\
\hline BBAS3 & 0,000872 & 0,000884 & $-0,429172$ & 7,845685 \\
\hline CMIG4 & 0,000795 & 0,000925 & 0,127131 & 6,964728 \\
\hline ALLL11 & 0,001546 & 0,000574 & 0,068112 & 0,360644 \\
\hline NETC4 & $-0,000665$ & 0,002292 & 0,152698 & 6,084733 \\
\hline CESP6 & 0,001310 & 0,000912 & $-0,918288$ & 9,591074 \\
\hline TNLP4 & 0,000336 & 0,000458 & 0,279915 & 2,315050 \\
\hline BRAP4 & 0,000972 & 0,000753 & $-0,032073$ & 2,519139 \\
\hline CYRE3 & 0,002809 & 0,001138 & 0,429855 & 1,981169 \\
\hline GOLL4 & 0,000972 & 0,000753 & $-0,032073$ & 2,519139 \\
\hline ANBEV4 & 0,001014 & 0,000488 & $-0,605079$ & 11,422060 \\
\hline BTOW3 & 0,001698 & 0,000912 & 0,347728 & 2,564047 \\
\hline TAMM4 & 0,000637 & 0,000740 & 0,100766 & 0,733885 \\
\hline LAME4 & 0,001082 & 0,001313 & 0,292131 & 3,891767 \\
\hline PRGA3 & 0,001051 & 0,000773 & 0,272220 & 3,828719 \\
\hline ELET6 & 0,000562 & 0,001096 & 0,412194 & 5,937587 \\
\hline
\end{tabular}


continua...

\begin{tabular}{|c|c|c|c|c|}
\hline LREN3 & 0,003808 & 0,001508 & 3,249326 & 36,967420 \\
\hline CSAN3 & 0,000545 & 0,001286 & $-0,130765$ & 0,885528 \\
\hline SDIA4 & 0,001304 & 0,000844 & 2,444551 & 66,083910 \\
\hline TCSL4 & 0,000568 & 0,000715 & 0,062971 & 0,583426 \\
\hline GFSA3 & 0,000772 & 0,000989 & 0,323113 & 1,071139 \\
\hline ELET3 & 0,000381 & 0,001122 & 0,328517 & 4,489243 \\
\hline NATU3 & 0,000973 & 0,000663 & 0,117048 & 2,197374 \\
\hline BRKM5 & 0,000418 & 0,000798 & 0,158037 & 2,917147 \\
\hline VIVO4 & $-0,000158$ & 0,001373 & 0,034374 & 5,803075 \\
\hline ARCZ6 & 0,000863 & 0,000800 & 1,393471 & 24,473840 \\
\hline ELPL6 & 0,000434 & 0,001497 & $-0,408577$ & 13,662190 \\
\hline CPLE6 & 0,000390 & 0,001083 & 0,371680 & 8,218264 \\
\hline GOAU4 & 0,001527 & 0,000906 & $-3,722023$ & 88,055100 \\
\hline DURA4 & 0,000849 & 0,000633 & 0,063737 & 2,926385 \\
\hline CCRO3 & 0,001441 & 0,000813 & $-0,478675$ & 10,484160 \\
\hline EMBR3 & 0,001184 & 0,001158 & 1,007968 & 11,959450 \\
\hline BRTO4 & 0,000526 & 0,000941 & $-0,116368$ & 4,618696 \\
\hline BRTP4 & 0,000580 & 0,000914 & 0,857440 & 9,341900 \\
\hline PCAR4 & 0,000691 & 0,000764 & 0,336426 & 15,300970 \\
\hline VCPA4 & 0,000989 & 0,000731 & 0,357681 & 5,471516 \\
\hline CPFE3 & $-0,000253$ & 0,000558 & 1,359586 & 3,643901 \\
\hline TNLP3 & 0,001122 & 0,001186 & 0,121851 & 4,997709 \\
\hline JBSS3 & 0,001297 & 0,001246 & 0,203836 & 1,080831 \\
\hline USIM3 & $-0,007025$ & 0,003720 & $-3,594482$ & 15,296050 \\
\hline RSID3 & 0,000564 & 0,016758 & $-0,182302$ & 24,300330 \\
\hline SBSP3 & 0,000495 & 0,001035 & $-1,142615$ & 18,354580 \\
\hline CRUZ3 & 0,001101 & 0,000598 & 0,178517 & 3,616139 \\
\hline UGPA4 & 0,001067 & 0,000354 & 0,018803 & 1,633068 \\
\hline KLBN4 & 0,000762 & 0,001032 & 0,543789 & 4,167142 \\
\hline BNCA3 & 0,000363 & 0,000751 & 1,518721 & 15,311350 \\
\hline TRPL4 & 0,001534 & 0,001145 & 0,516577 & 5,361028 \\
\hline BRTP3 & 0,001048 & 0,001243 & 1,136312 & 17,875440 \\
\hline TCSL3 & $-0,004039$ & 0,000887 & 0,018448 & 1,302081 \\
\hline LIGT3 & $-0,000218$ & 0,001423 & 2,667681 & 56,223680 \\
\hline TMAR5 & 0,000847 & 0,000598 & 0,155149 & 0,613022 \\
\hline TLPP4 & 0,000651 & 0,000710 & 1,345266 & 26,874540 \\
\hline CGAS5 & 0,000789 & 0,001126 & 0,473897 & 14,125170 \\
\hline TMCP4 & 0,000659 & 0,001189 & 0,388086 & 4,485096 \\
\hline CLSC6 & 0,000620 & 0,000952 & 0,095393 & 3,679304 \\
\hline CCPR3 & 0,002800 & 0,001072 & 1,963869 & 6,298166 \\
\hline
\end{tabular}


O retorno das ações possui assimetria negativa em 20 papéis e assimetria positiva em 47, o que significa que, em sua grande maioria, a moda e mediana do retorno é menor do que a média, havendo retorno normalmente menor do que o esperado.

Entre as dez ações mais líquidas do índice, a PETR4, BBDC4, USIM5, UBBR11, PETR3 e GGBR4 possuem assimetria negativa, ou seja, são interessantes investimentos porque possuem moda e mediana maiores do que o retorno esperado.

\subsection{Análise da estacionariedade das variáveis}

A tabela 3 apresenta a análise da estacionariedade das variáveis, isto é, se elas possuem mais de uma raiz.

Tabela 3 - Análise da estacionariedade das variáveis

\begin{tabular}{|l|c|c|}
\hline \multirow{2}{*}{ Ação } & \multicolumn{2}{|c|}{$\boldsymbol{R}$} \\
\cline { 2 - 3 } & ADF $\boldsymbol{p}$-vlr & KPSS $\boldsymbol{p}$-vlr \\
\hline PETR4 & 0,010 & 0,100 \\
\hline VALE5 & 0,010 & 0,100 \\
\hline BBDC4 & 0,010 & 0,100 \\
\hline VALE3 & 0,010 & 0,100 \\
\hline ITAU4 & 0,010 & 0,100 \\
\hline USIM5 & 0,010 & 0,090 \\
\hline UBBR11 & 0,010 & 0,100 \\
\hline CSNA3 & 0,010 & 0,100 \\
\hline PETR3 & 0,010 & 0,100 \\
\hline GGBR4 & 0,010 & 0,100 \\
\hline ITSA4 & 0,010 & 0,100 \\
\hline BBAS3 & 0,010 & 0,100 \\
\hline CMIG4 & 0,010 & 0,100 \\
\hline ALLL11 & 0,010 & 0,100 \\
\hline NETC4 & 0,010 & 0,100 \\
\hline CESP6 & 0,010 & 0,100 \\
\hline TNLP4 & 0,010 & 0,100 \\
\hline BRAP4 & 0,010 & 0,016 \\
\hline CYRE3 & 0,010 & 0,091 \\
\hline GOLL4 & 0,010 & 0,016 \\
\hline AMBV4 & 0,010 & 0,100 \\
\hline BTOW3 & 0,010 & 0,100 \\
\hline TAMM4 & 0,010 & 0,053 \\
\hline LAME4 & 0,010 & 0,065 \\
\hline PRGA3 & 0,010 & 0,100 \\
\hline ELET6 & 0,010 & 0,100 \\
\hline LREN3 & 0,010 & 0,100 \\
\hline CSAN3 & 0,010 & 0,100 \\
\hline SDIA4 & 0,010 & 0,100 \\
\hline TCSL4 & 0,010 & 0,100 \\
\hline GFSA3 & 0,010 & 0,100 \\
\hline ELET3 & 0,010 & 0,100 \\
\hline NATU3 & 0,010 & 0,069 \\
\hline BRKM5 & 0,010 & 0,100 \\
\hline VIVO4 & 0,010 & 0,100 \\
\hline & & \\
\hline & & 0 \\
\hline
\end{tabular}

Rev. Adm. UFSM, Santa Maria, v. 3, n. 3, p. 326-345, set./dez. 2010 


\begin{tabular}{|l|l|l|}
\hline ARCZ6 & 0,010 & 0,100 \\
\hline ELPL6 & 0,010 & 0,100 \\
\hline CPLE6 & 0,010 & 0,100 \\
\hline GOAU5 & 0,010 & 0,100 \\
\hline DURA4 & 0,010 & 0,100 \\
\hline CCRO3 & 0,010 & 0,100 \\
\hline EMBR3 & 0,010 & 0,081 \\
\hline BRTO4 & 0,010 & 0,100 \\
\hline BRTP4 & 0,010 & 0,100 \\
\hline PCAR4 & 0,010 & 0,100 \\
\hline VCPA4 & 0,010 & 0,100 \\
\hline CPFE3 & 0,066 & 0,100 \\
\hline TNLP3 & 0,010 & 0,100 \\
\hline JBSS3 & 0,010 & 0,100 \\
\hline USIM3 & 0,343 & 0,100 \\
\hline RSID3 & 0,010 & 0,100 \\
\hline SBSP3 & 0,010 & 0,100 \\
\hline CRUZ3 & 0,010 & 0,100 \\
\hline UCPA4 & 0,010 & 0,100 \\
\hline KLBN4 & 0,010 & 0,100 \\
\hline BNCA3 & 0,010 & 0,100 \\
\hline TRPL4 & 0,010 & 0,100 \\
\hline BRTP3 & 0,010 & 0,100 \\
\hline TCSL3 & 0,048 & 0,100 \\
\hline LIGT3 & 0,010 & 0,100 \\
\hline TMAR5 & 0,010 & 0,100 \\
\hline TLPP4 & 0,010 & 0,100 \\
\hline CGAS5 & 0,010 & 0,100 \\
\hline TMCP4 & 0,010 & 0,100 \\
\hline CLSC6 & 0,010 & 0,100 \\
\hline CCPR3 & 0,368 & 0,100 \\
\hline & & \\
\hline
\end{tabular}


No teste ADF, a hipótese nula é de raiz unitária, sendo a série estacionária se houver rejeição da hipótese nula, ou seja, o p-value menor do que 5\% para 95\% de confiança.

A maioria das ações possui estacionariedade do retorno, com exceção da série das ações da ELET3, CPFE3, USIM3 e CCPR3.

No teste confirmatório KPSS, que possui como hipótese nula a estacionariedade, portanto, para que a série seja estacionária, é preciso $p$-value maior do que $5 \%$ para uma confiança de 95\%. Com o teste de conformação KPSS, mais robusto, apenas as séries dos papéis da BRAP4 e da GOLL4 não são estacionárias.

\subsection{Medidas de dispersão das ações}

A tabela 4 apresenta as medidas de dispersão das ações em relação à proxy de mercado. Os valores de beta maiores do que 2,0 significam que a ação movimenta-se duas vezes com maior reação ou risco que o mercado. Se for 1,0, significa que se movimenta na mesma direção, ou seja, significa que possui a mesma reação ou risco que o mercado (isto é, risco médio). Se o valor for de 0,5, quer dizer que possui apenas a metade da reação ou risco que o mercado. Zero, se não for afetada pelos movimentos do mercado; - 0,5 a ação movimenta-se em apenas a metade da reação ou risco que o mercado; -1,0 o título movimenta-se na direção oposta ao mercado, apresentando a mesma reação ou risco que o mercado (isto é, risco médio) e -2,0 o papel possui reação ou visão duas vezes maior que o mercado.

Como se observa, a PETR4 é a ação mais sensível às variações de mercado, seguida da VALE5, ELET6, VIVO4 e CSNA3.

$\mathrm{Na}$ análise da assimetria, se esta for positiva, significa que possui uma moda e mediana menores do que o valor esperado, e portanto, há uma frequência maior de valores menores do que o valor esperado. E se negativa possui moda e mediana maiores do que a média. Como se observa a PETR4 e a VALE5 são as que possuem maior assimetria positiva.

Tabela 4 - Medidas de dispersão das ações em relação a proxy de mercado

\begin{tabular}{|l|l|r|r|}
\hline \multirow{2}{*}{ Ação } & \multicolumn{2}{|c|}{ Medidas de dispersão das ações estudadas em relação a Proxy de mercado } \\
\cline { 2 - 4 } & Beta & Assimetria Sistemática & Curtose Assistemática \\
\hline PETR4 & 2,9438620 & 7,2564200 & 5,9434420 \\
\hline VALE5 & 1,8594240 & 8,2323010 & 3,7461480 \\
\hline BBDC4 & 0,7929339 & $-0,6738994$ & 0,5685094 \\
\hline VALE3 & 0,5841564 & 1,4783805 & 0,7986563 \\
\hline ITAU4 & 0,3559887 & $-0,0398692$ & 0,5038793 \\
\hline USIM5 & 0,9413301 & $-0,6910617$ & 0,6062668 \\
\hline UBBR11 & 0,2388489 & 0,8784150 & 0,3386252 \\
\hline CSNA3 & 0,6987009 & $-0,1713599$ & 0,5470124 \\
\hline PETR3 & 0,8818299 & $-0,1197169$ & 0,8175448 \\
\hline GGBR4 & 0,9231151 & 0,1323996 & 0,8445746 \\
\hline ITSA4 & 0,3565832 & 0,1646518 & 0,6096263 \\
\hline BBAS3 & 0,6003821 & $-1,3652539$ & 0,2201896 \\
\hline CMIG4 & 1,0369470 & 0,8127920 & 1,0287520 \\
\hline ALL11 & 0,7174471 & 0,8274321 & 0,7313472 \\
\hline NETC4 & 0,6611668 & $-1,0780751$ & 0,1507631 \\
\hline CESP6 & 0,7768006 & 1,4247909 & 0,8014446 \\
\hline TNLP4 & 0,9032039 & 0,7466062 & 0,8508131 \\
\hline BRAP4 & 0,7270962 & 1,1262207 & 0,7573016 \\
\hline CYRE3 & 1,0699635 & 0,8373137 & 1,1064955 \\
\hline GOLL4 & 0,7270962 & 1,1262207 & 0,7573016 \\
\hline AMBV4 & 0,5753661 & 0,6934546 & 0,6628157 \\
\hline
\end{tabular}


continua...

\begin{tabular}{|c|c|c|c|}
\hline BTOW3 & 0,9337573 & 0,4649635 & 0,9465640 \\
\hline TAMM4 & 0,7555912 & 0,8414565 & 0,7751899 \\
\hline LAME4 & 0,5855011 & 0,1192187 & 0,4028644 \\
\hline PRGA3 & 0,5145589 & 0,6778259 & 0,6095342 \\
\hline ELET6 & 1,1727010 & 1,4188350 & 1,1408220 \\
\hline LREN3 & 0,9376851 & 1,0332297 & 0,9656673 \\
\hline CSAN3 & 1,1365090 & 1,6250820 & 1,1689700 \\
\hline SDIA4 & 0,1790387 & 0,3459392 & 0,1223537 \\
\hline TCSL4 & $-0,0715137$ & $-0,4954903$ & $-0,1464979$ \\
\hline GFSA3 & 0,9874165 & 0,9508452 & 1,0189196 \\
\hline ELET3 & 1,1341190 & 1,5273050 & 1,0274680 \\
\hline NATU3 & 0,6106810 & 0,5614524 & 0,6612279 \\
\hline BRKM5 & 0,6560713 & $-0,0793069$ & 0,5232602 \\
\hline VIVO4 & 1,1492840 & 1,0503850 & 1,0504690 \\
\hline ARCZ6 & 0,4344251 & 2,2255120 & 0,8137821 \\
\hline ELPL6 & 0,9203555 & $-0,4950229$ & 0,7035219 \\
\hline CPLE6 & 1,0103780 & 1,5411840 & 1,0994110 \\
\hline GOAU4 & 0,6617043 & $-0,6976614$ & 0,4434186 \\
\hline DURA4 & 0,4430471 & $-0,7700124$ & 0,3393432 \\
\hline CCRO3 & 0,5881223 & 0,9918891 & 0,6926622 \\
\hline EMBR3 & 0,6329491 & 0,4843716 & 0,5888562 \\
\hline BRTO4 & 0,8954632 & 0,0122663 & 0,7097933 \\
\hline BRTP4 & 1,0399210 & 1,3081570 & 1,1583490 \\
\hline PCAR4 & 0,6087249 & 0,6178588 & 0,9330484 \\
\hline VCPA4 & 0,5130794 & $-0,2243939$ & 0,5322518 \\
\hline CPFE3 & 0,9551446 & 1,5570183 & 1,3667755 \\
\hline TNLP3 & 1,1356979 & 0,7204134 & 0,8805755 \\
\hline BSS3 & 0,8794773 & 1,4154842 & 1,0300128 \\
\hline USIM3 & $-1,0281060$ & $-6,8237330$ & $-3,8306550$ \\
\hline RSID3 & 0,8983558 & $-0,3847199$ & 0,2115454 \\
\hline SBSP3 & 0,86668699 & $-1,0546713$ & 0,8401599 \\
\hline CRUZ3 & 0,5027080 & 0,1420693 & 0,3710468 \\
\hline UGPA4 & 0,3942655 & 0,7127437 & 0,4763328 \\
\hline KLBN4 & 0,6593709 & 0,1695862 & 0,5847256 \\
\hline BNCA3 & 0,7729255 & 1,1611321 & 0,9190391 \\
\hline TRPL4 & 1,0327150 & 1,6242740 & 1,0763960 \\
\hline BRTP3 & 1,1178050 & 1,7416690 & 1,5648450 \\
\hline TCSL3 & 1,0751420 & 1,4115420 & 1,1269110 \\
\hline LIGT3 & 0,8239249 & $-2,1352233$ & 0,3264502 \\
\hline TMAR5 & 0,9848362 & 0,5383244 & 0,9942524 \\
\hline TLPP4 & 0,8462748 & 1,5945816 & 1,3092618 \\
\hline CGAS5 & 0,7393869 & $-1,4762064$ & 0,5489926 \\
\hline TMCP4 & 0,8654583 & 0,1848476 & 0,4743594 \\
\hline CLSC6 & 0,8431993 & $-0,2466051$ & 0,6739476 \\
\hline CCPR3 & 0,9352029 & 0,5211310 & 0,3165517 \\
\hline
\end{tabular}


Na análise da curtose, se a ação possui curtose maior que 0,263, é platicúrtica, portanto, com caudas longas e achatadas. Se menor do que 0,263, significa que é leptocúrtica, ou seja, com caudas curtas e pontiagudas. As variáveis possuem, em sua totalidade, característica platicúrtica, mais uma vez apresentando maior sensibilidade as ações da PETR4 e da VALE5.

\subsection{Coeficientes estimados no relacionamento entre retorno esperado e suas medidas de dispersão}

A análise da relevância ou não dos indicadores de assimetria e curtose para entendimento dos dados dos ativos é investigada, inicialmente, em relação aos seus próprios valores. Na tabela 5, são apresentados os parâmetros estimados e seus respectivos testes de significância estatística.

Na parte superior da tabela é apresentado o prêmio pelo desvio-padrão quando este é utilizado como única variável explicativa. Nessa situação, o modelo não ajuda a explicar o comportamento do retorno das ações. A inclusão da assimetria no modelo melhora a explicação do modelo, atuando a favor do aumento da rentabilidade, da mesma forma que a inclusão do prêmio pela curtose. No entanto, o $\mathrm{R}^{2}$ ajustado em todos os modelos é muito baixo, o que significa que as medidas de dispersão não são bons parâmetros para explicar o retorno das ações em relação ao mercado.

Tabela 5 - Coeficientes estimados no relacionamento entre retorno esperado e suas medidas de dispersão

\begin{tabular}{|c|c|c|c|c|c|}
\hline $\begin{array}{l}\text { Modelo de } \\
\text { Mercado }\end{array}$ & Constante $b_{0}$ & $\begin{array}{c}\text { Prêmio pelo } \\
\text { Desvio Padrão } b_{1}\end{array}$ & $\begin{array}{c}\text { Prêmio pela } \\
\text { Assimetria } b_{2}\end{array}$ & $\begin{array}{l}\text { Prêmio pela } \\
\text { Curtose } b_{3}\end{array}$ & $\mathrm{R}^{2}$ Ajustado \\
\hline Modelo 1 & $\begin{array}{l}0.00732 * * \\
(0.0007430)\end{array}$ & $\begin{array}{c}0.82540 \\
(0.0012482)\end{array}$ & - & - & -0.01462 \\
\hline Modelo 2 & $\begin{array}{c}0.376 \\
(3.459 \mathrm{e}-04)\end{array}$ & $\begin{array}{c}0.814 \\
(1.322 \mathrm{e}-03)\end{array}$ & $\begin{array}{c}0.164 \\
(1.193 \mathrm{e}-05)\end{array}$ & - & 0.0005215 \\
\hline Modelo 3 & $\begin{array}{c}0.998 \\
(1.308 \mathrm{e}-06) \\
\end{array}$ & $\begin{array}{c}0.772 \\
(1.623 \mathrm{e}-03) \\
\end{array}$ & $\begin{array}{c}0.184 \\
(1.134 \mathrm{e}-05) \\
\end{array}$ & $\begin{array}{c}0.226 \\
(1.054 \mathrm{e}-05) \\
\end{array}$ & 0.008233 \\
\hline \multicolumn{6}{|c|}{$\begin{array}{ll}\text { Modelo } 1 & r_{i}=b_{0}+b_{1} \sigma_{i}+\varepsilon_{i,} i=1, \ldots, 66 \\
\text { Modelo } 2 & r_{i}=b_{0}+b_{1} \sigma_{i}+b_{2} S_{i}+\varepsilon_{i \prime} i=1, \ldots, 66 \\
\text { Modelo } 3 & r_{i}=b_{0}+b_{1} \sigma_{i}+b_{2} S_{i}+b_{3} k_{i}+\varepsilon_{i}, i=1, \ldots, 66\end{array}$} \\
\hline
\end{tabular}

\subsection{Coeficientes estimados para os modelos de mercado no período estudado}

Na tabela 6 são apresentados os coeficiente estimados pelos quatro modelos de mercado. Os coeficientes estimados são os prêmios associados pelos modelos para a variância, assimetria e curtose sistemática. 
Tabela 6 - Coeficientes estimados para os modelos de mercado no período estudado

\begin{tabular}{|c|c|c|c|c|c|}
\hline $\begin{array}{l}\text { Modelo de } \\
\text { Mercado }\end{array}$ & Constante $b_{0}$ & $\begin{array}{l}\text { Prêmio pela } \\
\text { variância } \\
\text { sistemática } b_{1}\end{array}$ & $\begin{array}{l}\text { Prêmio pela } \\
\text { Assimetria } \\
\text { sistemática } b_{2} \\
\end{array}$ & $\begin{array}{l}\text { Prêmio pela } \\
\text { Curtose } \\
\text { sistemática } b_{3}\end{array}$ & $\mathrm{R}^{2}$ Ajustado \\
\hline Modelo 1 & $\begin{array}{c}0.140 \\
(5.031 \mathrm{e}-04) \\
\end{array}$ & $\begin{array}{c}0.334 \\
(8.658 \mathrm{e}-06) \\
\end{array}$ & - & - & -0.0008123 \\
\hline Modelo 2 & $\begin{array}{c}0.263 \\
(4.427 \mathrm{e}-04) \\
\end{array}$ & $\begin{array}{c}0.443 \\
(7.502 \mathrm{e}-06) \\
\end{array}$ & $\begin{array}{c}0.760 \\
(2.954 \mathrm{e}-06) \\
\end{array}$ & - & -0.01518 \\
\hline Modelo 3 & $\begin{array}{c}0.256 \\
(4.534 \mathrm{e}-04) \\
\end{array}$ & $\begin{array}{c}0.811 \\
(3.416 \mathrm{e}-06) \\
\end{array}$ & $\begin{array}{c}0.964 \\
(-6.048 \mathrm{e}-07) \\
\end{array}$ & $\begin{array}{c}0.693 \\
(7.343 \mathrm{e}-06) \\
\end{array}$ & -0.02893 \\
\hline \multicolumn{6}{|c|}{$\begin{array}{ll}\text { Modelo } 1 & r_{i}=b_{0}+b_{1} \beta_{i}+\varepsilon_{i,} i=1, \ldots, 66 ; \\
\text { Modelo } 2 & r_{i}=b_{0}+b_{1} \beta_{i}+b_{2} Y_{i}+\varepsilon_{i,} i=1, \ldots, 66 ; \\
\text { Modelo } 3 & r_{i}=b_{0}+b_{1} \beta_{i}+b_{2} Y_{i}+b_{3} \delta_{i}+\varepsilon_{i,} i=1, \ldots, 66\end{array}$} \\
\hline
\end{tabular}

Agora procuram-se evidências de relacionamento entre retorno médio e suas co-oscilações com a proxy de mercado. Os coeficientes estimados pelos modelos que incorporam o terceiro e o quarto momentos (Modelos 2 e 3) não identificam nenhuma associação significativa com o retorno médio, variância, assimetria e curtose sistemática.

Portanto, constata-se que a inclusão do terceiro e quarto momentos no modelo básico de precificação não contribuem de maneira relevante para o entendimento de relacionamento entre as variáveis, risco e retorno, no processo de identificação do retorno médio das ações que compõem o índice Ibovespa.

\section{CONCLUSÃO}

Neste estudo, foi investigada a relevância da inclusão de informações de momentos superiores no modelo básico de precificação de ativos CAPM. Isto significa a inclusão de informações sobre a coassimetria e a cocurtose no processo de formação de retornos médios das ações que compõem o índice Ibovespa, considerando-se um índice igualmente ponderado dos 66 ativos que formaram o índice, em 31 de maio de 2008, como proxy de mercado.

Os resultados obtidos identificaram que existe excesso de curtose entre as variáveis que possuem características platicúrticas, comparadas a uma distribuição normal. Ou seja, as distribuições possuem caudas pesadas, como é comum em séries de ativos financeiros.

Os testes ADF e KPSS apontaram comportamento estacionário para as variáveis. Esse resultado torna confiável a inferência sobre os parâmetros estudados com base nos testes $t, F$ e no coeficiente de determinação. Os parâmetros estimados para o desvio padrão, assimetria e curtose foram significativos. O modelo que inclui todos os fatores é o que se apresenta como mais significativo da rentabilidade média das ações. No entanto, apesar dos prêmios pelo desvio padrão, assimetria e curtose serem positivos, o coeficiente de determinação ajustado possui um valor muito baixo para os paradigmas econômicos.

$\mathrm{Na}$ análise dos resultados da relação entre os retornos médios com as variáveis variância sistemática, assimetria sistemática e curtose sistemática pode-se afirmar que a inclusão das variáveis de momentos superiores melhora o desempenho do modelo básico que considera apenas a variância sistemática, porém o $R^{2}$ Ajustado continua apresentando valores muito baixos para sua aplicação econômica, o que sustenta a teoria do modelo de índice único de Sharpe (1964). 


\section{REFERÊNCIAS BIBLIOGRÁFICAS}

ARDITI, F. D. Risk and the required return on equity. Journal of Finance, v. 22, n. 1, p. 19-36, 1967.

BERENYI, Z. Measuring hedge fund risk with multimoment risk measures. Working paper, 2002.

CATARINA, S. F. G.; CERETTA, P. S.; MULLER, I. Precificação de ativos incorporando momentos superiores. Workpaper, 2007.

CHRISTIE-DAVID, R., CHAUDRY, M. Coskewness and cokurtosis in futures markets. Journal of Empirical Finance, v.8, p.55-81, 2001.

CHUNG, P., JOHNSON, H., SCHILL M. Asset pricing when returns are non normal: FamaFrench factors vs higher order systematic comoments. Working paper, 2001.

CUNHACHINDA, P.; DANDAPANI, K.; HAMID, S.; PRAKASH, A. J. Portfólio selection and skewness: evidence from international stock markets. Jounal of Banking and Finance, v. 21, p. 143-343, 1997.

FANG, H. LAI, T. Y. Cokurtose and capital asset pricing. The Financial Review, v. 32, n. 2, p. $427-$ 465, 1999.

GAMBA, A.; ROSSI, F. A. A three-moment based capital asset pricing model. In: WORKSHOP ON CONVEXITY AND PARALLEL COMPUTING, 1997, Verona. Proceeding... Verona: University of Verona, 1997. p. 113-129.

GAMBA, A., ROSSI, F. Mean-variance-skewness analysis in portfolio choice and capital markets. Working paper, 1998.

GROPELLI, A. A.; NIKBAKHT, E. Administração financeira. 2. ed. São Paulo: Saraiva, 2005.

HARVEY, C. The drivers of expected returns in international markets. Working paper, 2000.

HARVEY, C.; SIDDIQUE, A. Conditional skewness in asset pricing tests. Journal of Finance, v.3, p.12631295, jun. 2000.

HWANG, S.; SATCHELL, S. Modeling emerging market risk premia using higher moments. Working paper, 1999.
INGERSOLL, J. Multidimesional security pricing, Journal of Financial and Quantitative Analysis, v. 10, p.785-798, 1975.

JURCENZKO, E., MAILLET, B. The four-moment capital asset pricing model: some basic results. Working paper, 2002.

KRAUS, A., LITZENBERGER, R. Skewness preference and the valuation of risky assets, Journal of Finance, v. 31, p.1085-1100, 1976.

LIM, K. G. A new test of the three moment capital asset princing model. Journal of Financial and Quantitative Analysis, v. 31, p. 205-216, Jun. 1989.

LO, A. Risk management for hedge funds: introduction and overview. Working paper, 2001.

PEIRÓ, A. Skewness in financial returns. Journal of Banking and Finance, v. 23, p. 847-862, 1999.

RANALDO, A.; FAVRE, L. How to price hedge funds: from two- to four-moment CAPM. Edhec Risk And Asset Management Research Center. Disponível em: <http://www.fmpm.ch/docs/7th/ Papers_SGF_2004/SGF720.pdf.> Acesso em: 18 jun. 2007.

ROSS, S. A.; WESTERFIELD, R. W.; JAFFE, J. F. Administração financeira. São Paulo: Atlas, 1995.

RUBINSTEIN, M. The fundamental theorem of parameter preference security valuation. Journal of Financial and Quantitative Analysis, v. 8, p. 6169, Jan. 1973.

SARTORIS, A. Estatística e introdução à econometria. São Paulo: Saraiva, 2003.

SHARPE, W. Capital asset prices: a theory of market equilibrium under conditions of risk. The Journal of Finance, v. 19, n. 3, p. 425-442, sep. 1964.

VERGARA, S. C. Projetos e relatórios de pesquisa em administração. São Paulo: Atlas, 2000. 